\title{
Eqüidade na alocação de recursos para a saúde: a experiência do Estado do Rio Grande do Sul, Brasil
}

\author{
Equity in health resources allocation: \\ the experience of Rio Grande do Sul, Brazil
}

Maria Lecticia Machry de Pelegrini 1

Janice Dornelles de Castro 1

Maria de Lourdes Drachler 1

\footnotetext{
1 Universidade do Vale do Rio dos Sinos,

Unisinos, São Leopoldo.

Rua Eudoro Berlink 1.084/

201, Bairro Auxiliadora, 90450-030,

Porto Alegre RS.

mlmpelegrini@terra.com.br
}

Abstract The article analyses the capacity to distribute resources for health services, established by the policy of Municipalização Solidária da Saúde (MSS), created by the Health Department of the State of Rio Grande do Sul (SES/RS). The objective of this policy is to allocate resources to local level using a formula that would consider the equity criteria (the population and the $\mathrm{Hu}$ man Development Index - HDI), the period analyzed is the year of 2001. If the formula were adopted, a displacement of financial resources for cities with smaller population would be verified. In the usual way of financing health in Brazil (per capita or production) the resources would be driven to the cities with bigger population. To evaluate if the MSS policy benefits the cities in a worse social and economical condition, the HDI was used. In the State of the Rio Grande do Sul this index varies only from medium to high. There is no city with the low level of this indicator. Notwithstanding, the formula showed a great capacity to benefit cities in worse social and economical condition. Using the formula, half of the resources would go for $34 \%$ of the resident population in the cities with the lower HDI.

Key words Equity, Public health, Health economics, Health financing, Resources distribution
Resumo O artigo analisa a distribuição de recursos, estabelecida pela política de Municipalização Solidária da Saúde (MSS) da Secretaria de Estado da Saúde do Rio Grande do Sul (SES/RS) ao examinar sua potencialidade de desconcentrar recursos, conforme o tamanho da população residente e o nivel de desenvolvimento humano municipal, o período analisado foi o ano de 2001. A partir da adoção dos critérios, verifica-se um deslocamento de recursos financeiros para municípios com menor concentração populacional que obtiveram em seus Fundos Municipais de Saúde recursos em valores até então não captados pelas outras formas de financiamento da saúde, ocorrendo uma desconcentração de recursos. Para avaliar se a política da MSS beneficiou os municípios em piores condições socioeconômicas, utilizou-se o Índice de Desenvolvimento Humano - Municipal (IDHM), que no Rio Grande do Sul apresenta pequenas variações (apenas municípios com IDH-M médio e alto. Mesmo assim, verificou-se que a política de MSS prevê a distribuição de metade dos recursos para $34 \%$ da população dos municípios com os menores índices do IDH-M, apontando a grande capacidade desta política para a desconcentração de recursos aos municípios que necessitam ter potencializados seus serviços de saúde para a melhoria das condições de vida da população. Palavras-chave Eqüidade, Saúde coletiva, Economia da saúde, Financiamento da saúde, Distribuição de recursos 


\section{Introdução}

O financiamento de políticas públicas e, especificamente, das políticas de saúde tem sido foco de interesse e pesquisa em todo o mundo, especialmente após a II Guerra Mundial, quando o provimento dos cuidados e serviços de saúde torna-se atribuição de Estado em muitos países, e a saúde passa a ser entendida como um direito universal, isto é, estendida a todos, como atributo da cidadania (Médici A, 2002).

A partir das décadas de 1950 e 1960, os gastos públicos nessa área cresceram de maneira considerável, especialmente em países desenvolvidos, seja pelo aumento de cobertura dos serviços, pela evolução e incorporação de novas tecnologias, ou pelo aumento da esperança de vida da população. Neste contexto, a discussão sobre o financiamento do setor ocupa a pauta de governantes e economistas nos países europeus, com destaque ao governo britânico, que desenvolve processo de acompanhamento de alocação eqüitativa de recursos para saúde desde os anos 70. Além das experiências européias, também há exemplos na Âfrica do Sul e Nova Guiné, entre outros $\mathrm{O}$ grande desafio é o desenvolvimento de mecanismos de alocação que venham garantir a eficiência e a eqüidade no financiamento das políticas e ações de saúde.

No Brasil, o direito universal e integral à saúde foi conquistado pela sociedade na Constituição de 1988, com a criação do Sistema Único de Saúde (SUS). A Constituição, além de afirmar a saúde como direito de todos e dever do Estado, assegura a sua universalidade e integralidade com o financiamento tripartite (União, Estados e Municípios) e com diversidade de fontes. Estes preceitos constitucionais encontram-se reafirmados na Lei Orgânica da Saúde de 1990 (Brasil, 1990). No entanto, o financiamento do sistema tem sido um dos grandes desafios, pois é contraditório com o modelo econômico dominante no País desde a década de 1990, caracterizado pelo estabelecimento do "estado mínimo", de uma relação ainda muito verticalizada entre as três esferas de governo e pela ausência de políticas públicas voltadas para a atenção integralizada à população. Além disso, o modelo centralizado até então existente na condução da política de saúde brasileira e o modelo assistencial centrado na doença e em procedimentos médicos geram respostas inadequadas ou insuficientes aos resultados anunciados na legislação em vigor e distorções na forma de financiamento da saúde, ainda re- lacionada em grande medida com a oferta e a produção de serviços.

A década de 1990 foi promissora na ampliação de sistemas municipais de saúde. No entanto, observa-se que as esferas estaduais mantiveram-se longe de adequar suas estruturas a fim de cumprir as obrigações constitucionais, especialmente no que tange ao previsto no inciso III do artigo 17o da Lei Orgânica da Saúde (lei federal no 8.080/90): "prestar apoio técnico e financeiro aos municípios” (Brasil, 1990).

No Rio Grande do Sul, o governo do período de 1999-2002 implantou a Municipalização Solidária da Saúde, política de cooperação técnica e financeira que, para fortalecer a descentralização do sistema de saúde prevista constitucionalmente, estabeleceu critérios para repassar recursos do tesouro estadual aos municípios. A esses critérios foi associada a capacidade de promover eqüidade.

Essa política de financiamento foi premiada no Concurso Nacional de Experiências Inovadoras do SUS, realizada pelo Ministério da Saúde no ano 2000, por suas características inovadoras (Ministério da Saúde, 2002). Segundo a análise dos avaliadores, mereciam destaque os seguintes aspectos: garantir a participação efetiva da esfera estadual no financiamento do SUS, com repasses diretos do fundo estadual para os fundos municipais de saúde; garantir a autonomia dos municípios para a definição da aplicação dos recursos através do gestor municipal e com o controle social; e utilizar critérios de eqüidade para calcular o valor destinado aos municípios, conforme previsto no artigo $35 \mathrm{da}$ Lei Orgânica da Saúde.

Neste artigo, analisaremos se o uso de critérios que buscam garantir maior eqüidade na distribuição de recursos financeiros propostos pela Política de Municipalização Solidária no Rio Grande do Sul possui, de fato, esta capacidade redistributiva, e afeta positivamente as desigualdades apontadas pelo Índice de Desenvolvimento Humano Municipal (IDH-M). Também compõe o escopo dos objetivos deste trabalho a formulação de hipóteses capazes de desafiar novos estudos sobre efeitos intangíveis pela metodologia de estudo adotada, inclusive acerca da capacidade deste indicador subsidiar adequadamente o planejamento do sistema de saúde num contexto de uma população com as características que tem atualmente o Estado do Rio Grande do Sul, quais sejam: aumento da população acima de 60 anos, grande concentração de municípios de pequeno porte $(68 \%$ 
dos municípios possuem população até 10.000 habitantes).

\section{A busca de eqüidade no financiamento}

A busca de eqüidade vem desafiando gestores e planejadores de sistemas de saúde há algumas décadas. Os esforços nacionais para diminuir as iniqüidades em saúde vêm sendo desenvolvidos, conforme aponta Tobar (2003), em três dimensões básicas:

1. Eqüidade na dotação de recursos financeiros: toda população deve receber os recursos adequados para dar resposta as suas necessidades (fazendo-se aqui toda a diferenciação entre o que é igualitário e o que é justo).

2. Eqüidade no acesso a serviços: todas as pessoas devem receber atenção integral em todo e qualquer episódio de doença ou risco.

3. Eqüidade nos resultados: todos os setores da sociedade, independente de suas condições sociais e econômicas, devem alcançar resultados lineares em indicadores de saúde, medidos por indicadores clássicos, como esperança de vida ao nascer e taxas de morbi-mortalidade.

O princípio de eqüidade reconhece que os indivíduos são diferentes entre si e merecem, portanto, tratamento diferenciado que elimine ou reduza a desigualdade. Ou seja, o tratamento desigual é justo quando é benéfico ao indivíduo mais carente (Vianna et al., 2001).

Cabe salientar que as desigualdades em saúde refletem desigualdades sociais e que a igualdade no acesso e uso dos serviços de saúde não é suficiente para diminuir as desigualdades no adoecer e morrer existentes entre grupos sociais.

Essa distinção é importante no desenho de políticas públicas para que possamos trabalhar com a noção de necessidades diferentes entre distintos grupos sociais, determinadas tanto pela diversidade (sexo, idade, raça) como pela injustiça social (renda, acesso a bens e serviços) ou mesmo por características culturais e subjetivas de grupos e indivíduos.

Na reflexão sobre a forma mais justa de distribuir recursos em saúde, diversos autores (Almeida, 2002; Dachs, 2001; Drane, 1990; Giraldes, 1997; Medeiros, 1999; Sen, 2002a; 2002b; Tobar 2003; Porto, 2002; Barros, 1997) apontam que a sociedade é justa quando assegura ao cidadão a satisfação de suas necessidades por ações e serviços de saúde. Os países que adotam os sistemas de saúde de acesso universal, como o Brasil, entre eles a Inglaterra, Canadá e Austrália, reconhecem que, quando se trata de atenção à saúde, a escolha de pessoas ou grupos que devem merecer a prioridade na atenção do Estado não é óbvia. Isto porque há imperfeições na oferta de ações e serviços na área da saúde; a população em geral não tem possibilidade de custear diretamente todos os serviços de saúde que necessita e há uma impossibilidade de discriminar quem pode ou não se valer dos mesmos (Castro, 2002). Assim, é necessária a interferência do Estado para estabelecer os níveis adequados de produção e consumo desses serviços.

A busca de políticas de financiamento que propiciem a eqüidade teve como precursor o sistema britânico. Na década de 1970 foi elaborada a metodologia RAWP - Resource Allocation Working Party, que distinguiu diferentes critérios para orientar os gastos em saúde (de custeio e de investimentos), buscando assegurar que os recursos de investimentos conseguissem igualar as condições de acesso aos serviços de saúde nas diferentes regiões. Para a distribuição de recursos de custeio foram determinados critérios de necessidades regionais que incluem população ponderada pela taxa nacional de utilização dos serviços, pela idade e sexo e pela taxa de mortalidade regional padronizada (Castro, 1991; Côrtes et al. In: Ferla \& Fagundes, 2003). Desde a sua implantação, muitos ajustes já foram implementados, permanecendo como um dos principais métodos de avaliação sistêmica de necessidades. Este método tem sido base para a orientação de diversos outros países no Reino Unido: Escócia, Irlanda do Norte e País de Gales; demais países da Europa: Espanha e Portugal; África: Zâmbia e África do Sul; na América: Canadá, México e Brasil; e, Austrália, Nova Zelândia, Índia (Castro, 1991; Tobar, 2003).

Recentemente, as discussões no mundo sobre eqüidade na organização dos sistemas de saúde e nas políticas de financiamento para o setor vêm sofrendo o impacto de uma nova perspectiva. Além dos aspectos clássicos da disponibilidade de recursos financeiros, acesso a serviços de saúde e os resultados mais imediatos da atenção, vem tomando corpo um conjunto de estudos que contemplem a análise de necessidades de longo prazo no planejamento do sistema de saúde e, por decorrência, para a alocação de recursos financeiros. Por volta do final da década de 1990, esse movimento apontava a importância emergente da violência no impac- 
to sobre as necessidades de saúde (Briceño-León et al., 2000). No final de 2003, a publicação dos primeiros resultados de um estudo multicêntrico coordenado pela Organização Pan-Americana da Saúde (Opas) apontava conseqüências em curto prazo, para os sistemas de saúde da América Latina, do rápido envelhecimento da população e da diminuição das taxas de fecundidade verificados, principalmente a partir do final da década de 1970 (Lebrão et al., 2003).

No Brasil, a Lei Orgânica da Saúde (LOS 8.080/90) que, conforme o previsto na Constituição, dispõe sobre princípios e diretrizes para a promoção, proteção e recuperação da saúde, a organização e o funcionamento dos serviços correspondentes, registra alguns conceitos que no território nacional compuseram o escopo de um amplo movimento social na construção do que se convencionou denominar de processo de Reforma Sanitária brasileira: a universalidade de acesso aos serviços de saúde em todos os níveis de assistência, igualdade da assistência à saúde, sem preconceitos ou privilégios de qualquer espécie, a utilização da epidemiologia para o estabelecimento de prioridades, na alocação de recursos e a orientação programática, conjugação dos recursos financeiros, tecnológicos, materiais e humanos da União, Estados, do Distrito Federal e dos Municípios na prestação de serviços de assistência à saúde da população.

Estabelece ainda, em seu artigo 35, os critérios de financiamento do Sistema Único de Saúde, no qual a distribuição de recursos deva ser feita a partir da combinação de critérios que contemplem: perfil demográfico da região; perfil epidemiológico da população a ser coberta; características quantitativas e qualitativas da rede de saúde na área; desempenhos técnico, econômico e financeiro no período anterior; níveis de participação do setor saúde nos orçamentos estaduais e municipais; previsão do plano qüinqüenal de investimentos da rede; ressarcimento do atendimento a serviços prestados para outras esferas de governo (Brasil, 1990). A partir do disposto na legislação, o desafio tem sido a operacionalização do financiamento nas políticas de saúde implantadas, através das Normas Operacionais Básicas (NOB 91, NOB 93, NOB 96). O Ministério da Saúde tem apresentado políticas que representam retrocessos, como o predomínio da lógica de financiamento por produção, e também políticas que são avanços como a implantação do Piso de Atenção Básica (PAB), apesar dos baixos valores pagos aos municípios pelo $\mathrm{PAB}$ fixo, bem como o condicionamento do pagamento do $\mathrm{PAB}$ variável à adoção de programas específicos. Os demais integrantes do custeio em saúde - média e alta complexidade - continuam sendo financiados pelo pagamento de produção e sujeitos a oferta de serviços existentes, o que dificulta em muito a efetiva descentralização de serviços de saúde (Barros, 2003; Carvalho, 2002; Mendes \& Marques, 2002; Faveret, 2003; Ugá, 2003;Vianna, 1991; Jaeger, 2002).

A busca de critérios para a operacionalização da distribuição de recursos tem merecido a atenção da economia da saúde. O primeiro estudo realizado sobre o tema no Brasil foi feito no início da década de 1990 por Castro (1991) em uma discussão sobre os critérios de financiamento da manutenção da rede ambulatorial do Rio Grande do Sul, mostrando desta forma a relevância do tema e a preocupação dos atores envolvidos com o financiamento da saúde desde então.

Um dos grandes problemas encontrados para o desenvolvimento de metodologias alocativas de recursos é a dependência da existência de sistemas de informações integrados, confiáveis, abrangentes e acessíveis, que possibilitem a análise das desigualdades em saúde em diferentes dimensões (Viana, 2001). Para a operacionalização de indicadores relativos aos gastos em saúde, avanços já estão sendo feitos por meio do Sistema de Informações sobre Orçamentos Públicos em Saúde - SIOPS, que tem como objetivo contribuir para a orientação das políticas de saúde locais (Teixeira, 2003), constituindo-se em valioso instrumento de apoio à gestão e ao controle social. Para o bom funcionamento desse sistema, implantado em 1999, é necessária a sensibilização dos gestores no reconhecimento da necessidade de obter informações para formulação, implantação, acompanhamento e avaliação da política de saúde. Pastrana e Cunha (2002), em trabalho apresentado no Congresso da Associação Brasileira de Economia da Saúde, realizado em dezembro 2002, mostram, em estudo comparativo entre o Rio de Janeiro e o Rio Grande do Sul, que existe um padrão de alimentação dos dados do sistema diferenciado, com uma maior quantidade e qualidade dos dados dos municípios gaúchos. Podemos supor que esta diferença foi resultado da exigência do preenchimento destas informações para o cálculo do repasse de recursos estaduais no Rio Grande do Sul. 


\section{A experiência gaúcha}

A pequena participação dos Estados no financiamento do Sistema Único de Saúde tem sido apontada como um dos estrangulamentos do sistema. Estudo realizado sobre o tema, no Rio Grande do Sul, analisando o período de 1995 a 2001 (Ferla, 2002), constatou uma acentuada variação nos valores repassados pelo gestor estadual aos municípios e regiões, sendo que 30\% dos municípios não haviam recebido qualquer recurso financeiro estadual para a saúde até o ano de 1988, independente da modalidade de habilitação em que se encontravam ou de indicadores demográficos e de saúde. Essa característica da participação estadual no financiamento não fortaleceu a descentralização e hierarquização dos serviços, buscadas na regionalização ordenada do SUS, já que regiões com menor oferta de serviços, tiveram menores valores repassados pelo Estado.

Na gestão de 1999 a 2002, a Secretaria Estadual de Saúde (SES) estabelece, em seus eixos prioritários de atuação, a descentralização da gestão, criando a política de Municipalização Solidária da Saúde (MSS), através do decreto estadual no 30.582, de 10 de junho de 1999, que dispõe sobre o repasse regular e automático de recursos do fundo estadual de saúde para os fundos municipais de saúde.

Criada com o objetivo de fortalecer a gestão municipal, nessa política os recursos do Tesouro Estadual são transferidos aos municípios, sem qualquer vinculação com modalidades previamente definidas de gasto (Jaeger, 1999), dando aos mesmos autonomia para decidir o destino do recurso, desde que sua aplicação seja aprovada pelo respectivo Conselho de Saúde e o gasto seja realizado em projetos e atividades classificadas na função saúde.

No ano de 1999, os recursos foram distribuídos a partir do cálculo do valor per capita. No ano seguinte, além do perfil demográfico, foram pactuados outros critérios no Conselho Estadual de Saúde que atendiam a outras necessidades em saúde, tais como: perfil epidemiológico, características quantitativas e qualitativas da rede municipal, necessidades em saúde - perfil etário da população.

Então, a partir do ano de 2001, a base de cálculo dos recursos ficou assim constituída:

1. O Coeficiente de População Total do Município $(\mathrm{C} 1)$, calculado pela divisão do número total de habitantes do município no ano de 2001, ajustado para o universo dos 497 muni- cípios, segundo dados oficiais do Instituto Brasileiro de Geografia e Estatística (IBGE) publicados na Resolução no 07, de 15/8/2001, pelo número total de habitantes do Estado do Rio Grande do Sul no mesmo período, identificado pela mesma fonte.

2. O Coeficiente de População com Idade Menor de 14 Anos (C2), calculado pela divisão do número estimado de habitantes com idade menor de 14 anos do município no ano de 2001, segundo dados oficiais do IBGE ajustados para o ano de 2001 para o universo de 497 municípios, pelo número total estimado de habitantes com idade menor de 14 anos do Estado do Rio Grande do Sul no mesmo período, identificado pela mesma fonte.

3. O Coeficiente de População com Idade Maior de 60 Anos (C3), calculado pela divisão do número estimado de habitantes com idade maior de 60 anos do município no ano de 2001, segundo dados oficiais do IBGE ajustados para o ano de 2001 para o universo de 497 municípios, pelo número total estimado de habitantes com idade menor de 14 anos do Estado do Rio Grande do Sul no mesmo período, identificado pela mesma fonte.

4. O Coeficiente do Inverso da Capacidade Instalada Municipal (C4), calculado pelo inverso da divisão do número de consultórios cadastrados no município no Sistema de Informações Ambulatoriais (SIA/SUS), no mês de novembro de 2000 pelo número total de consultórios cadastrados no SIA/SUS no Estado do Rio Grande do Sul no mesmo período.

5. O Coeficiente Ponderado de Mortalidade Infantil do Município (C5), calculado pela divisão do peso atribuído ao Coeficiente de Mortalidade Infantil (CMI) do município nos anos de 1998 a 2000 pela soma total dos pesos atribuídos a todos os municípios do Estado.

6. O Coeficiente do Inverso da Receita Própria Municipal (C6), calculado a partir dos dados de arrecadação vinculável per capita, apurados pelo Sistema de Informações em Orçamentos Públicos em Saúde (Siops) relativamente aos valores médios dos anos-base de 1998 a 2000.

7. O Coeficiente do Percentual de Recursos Próprios Utilizados em Saúde (C7), calculado a partir dos dados de recursos próprios aplicados em saúde apurados pelo Sistema de Informações em Orçamentos Públicos em Saúde (Siops) relativo aos valores médios nos anosbase de 1998 a 2000.

8. O critério de prioridade regional foi calculado por um coeficiente (C8) criado pela divi- 
são do peso atribuído à ordem de prioridade dada à saúde pela população de cada município nas assembléias do Orçamento Participativo Estadual no ano de 2001.

Pela aplicação dos critérios descritos, cada município teve calculado um Coeficiente Geral pela seguinte fórmula:

$\mathrm{CG}=0,3(\mathrm{C} 1)+0,1(\mathrm{C} 2)+0,1(\mathrm{C} 3)+0,05(\mathrm{C} 4)+$ $0,05(\mathrm{C} 5)+0,05(\mathrm{C} 6)+0,05(\mathrm{C} 7)+0,3(\mathrm{C} 8)$

A partir dessa fórmula, aplicaram-se todos os critérios apontados no artigo 35 da Lei Orgânica da Saúde (Brasil, 1990), obteve-se uma proposta de distribuição de recursos capaz de realizar uma grande redistribuição, beneficiando especialmente os pequenos municípios que com a utilização desta fórmula tiveram os valores previstos de repasse muito maiores que se tivesse sido utilizado o critério per capita.

\section{A política de Municipalização \\ Solidária da Saúde - RS e a sua capacidade de desconcentrar recursos: análise do tamanho da população residente e do nível de desenvolvimento humano municipal}

Este é um estudo de análise econômica, que investiga a distribuição de recursos proposta pela política de Municipalização Solidária da Saúde para o ano de 2001 pela SES/RS, examinando a capacidade de desconcentrar recursos sobre duas variáveis independentes: o tamanho da população residente e o nível de desenvolvimento humano municipal.

Neste estudo foram incluídos todos os municípios do Estado do Rio Grande do Sul ( $\mathrm{N}=$ 497) existentes em 2001. Os dados referentes à população e aos valores previstos para repasse aos municípios através da MSS foram obtidos na portaria 26/2001 que estabeleceu o valor total de recursos, os critérios de distribuição e os dados populacionais, conforme IBGE.

Para examinar a capacidade de descentralizar recursos do método proposto pela política da MSS, as seguintes variáveis foram selecionadas: (a) valor previsto de repasse a cada município usando o método da MSS para o ano de 2001, (b) população dos municípios conforme o IBGE, (c) Índice de Desenvolvimento Humano Municipal (IDH-M) desenvolvido pelas $\mathrm{Na}$ ções Unidas.

O Rio Grande do Sul tinha, em 2001, uma população de 10.082.133 habitantes. O valor total previsto para repasse pela política MSS para o Estado naquele ano foi $\mathrm{R} \$ 64.930 .232,56$. Se a distribuição de recursos se baseasse simplesmente em um critério populacional, cada município do Estado receberia $\mathrm{R} \$ 6,44$ per capita. É importante salientar que não está sendo avaliada a potencialidade de promover a igualdade na distribuição dos recursos pela política de Municipalização Solidária da Saúde, que seria obtida pela distribuição per capita. Pretendese, no entanto, investigar a capacidade de promover a eqüidade, ou seja, garantir mais aos que precisam mais.

A distribuição, após a aplicação da metodologia da MSS, apresentou os seguintes resultados: (a) 76,46\% dos municípios perceberam acima da média de $\mathrm{R} \$ 6,44$ per capita; (b) $\mathrm{R} \$ 186,34$ foi o maior valor per capita e (c) R $\$ 1,60$ foi o menor valor per capita. Podemos inferir dessa informação que a MSS possui uma grande capacidade redistributiva, pois a maior parcela dos municípios teve os valores previstos de repasse majorados em relação a uma distribuição meramente igualitária (ou per capita). No entanto, utilizando a MSS, e após, fazendo o cálculo do valor per capita, observamos que a amplitude desses valores é ainda muito grande; por isso, resta analisar se essa distribuição foi equânime, para tanto foi utilizada a curva proposta por Lorenz, que é a representação gráfica da distribuição de determinado atributo (Hoffmann, 1998).

A curva de Lorenz tem sido usada para verificar a distribuição de financiamento e prestação de cuidados em saúde (Vianna, 2002). Neste caso, queremos medir como foram distribuídos os recursos previstos pela MSS para os municípios, em relação ao seu tamanho e ao seu Índice de Desenvolvimento Humano. Considerou-se o porte dos municípios como um elemento na análise das necessidades de saúde, visto que, historicamente, as políticas de financiamento concentram recursos e capacidade instalada em municípios com grandes aglomerados populacionais. Para a construção da curva, neste caso, os municípios foram colocados em ordem crescente de população. E para a construção da curva de Lorenz, buscando avaliar a qualidade de vida ou de desigualdade social, os municípios foram ordenados em função de seu Índice de Desenvolvimento Humano Municipal que mede o nível de desenvolvimento em três dimensões: renda, educação e longevidade.

$\mathrm{Na}$ Curva de Lorenz, a distribuição que expressa a perfeita igualdade forma uma linha de $45^{\circ}$, tendo no eixo $\mathrm{Y}$ os recursos previstos acumulados e no eixo $\mathrm{X}$ a população acumulada 
(Hoffmann, 1998). Nesse trabalho, os recursos previstos, apresentados no eixo $\mathrm{Y}$, foram reduzidos a um coeficiente variando de 0 a 1 , onde 1 representa a totalidade. No eixo $\mathrm{X}$ foi representada a população dos municípios gaúchos, também em coeficientes variando de 0 a 1, ordenados de duas maneiras, primeiro por (a) por ordem crescente de população dos municípios e (b) pelo Índice de Desenvolvimento $\mathrm{Hu}$ mano Municipal, também em ordem crescente de valores, como já especificado.

\section{Análise em relação ao tamanho da população residente}

Os resultados encontrados da relação entre as variáveis, recursos previstos e população acumulada, quando os municípios foram dispostos em ordem crescente de população, estão expressos no gráfico 1 . Pode-se verificar que se formou uma concavidade em relação à linha, demonstrando que foi obtida uma desigualdade na distribuição de recursos e essa desigualdade atendeu aos municípios de menor porte, os quais considerou-se como os que mais precisavam de recursos para atender às necessidades de saúde de sua população, demonstrando que a metodologia de distribuição proposta pela política de Municipalização Solidária da Saúde foi bem sucedida em garantir a eqüidade.
A curva resultante assume uma configuração que indica uma forte concentração de recursos (em torno de $70 \%$ dos recursos previstos), no segmento anterior à marca de $50 \%$ da população. Neste grupo encontram-se 376 municípios do universo de 497 existentes, indicando a concentração de recursos em municípios de pequeno porte.

Para analisar com maior detalhe o comportamento da distribuição dos recursos em relação à população, trabalhamos com as seguintes categorias: (a) municípios até 10.000 habitantes ( $\mathrm{N}=338$ municípios); (b) municípios entre 10.000 e 100.000 habitantes $(\mathrm{N}=142$ municípios); (c) municípios acima de 100.000 habitantes ( $\mathrm{N}=17$ municípios).

Os municípios até 10.000 habitantes representam $68 \%$ dos municípios gaúchos, possuem $14,28 \%$ da população do Estado e, na distribuição de recursos prevista, recebem $44,22 \%$ dos recursos. Já os municípios de médio porte, neste trabalho aqueles com população entre 10.000 e 100.000 habitantes, são $28,6 \%$ do total dos municípios, correspondendo a $41,06 \%$ da população e recebendo $32,54 \%$ dos recursos previstos. Apenas 3,4\% dos municípios têm mais de 100.000 habitantes estes, representam 44\% do total da população e obtiveram 23,24\% dos recursos previstos.

Para analisar em maior detalhe como foi a distribuição dos recursos, em cada uma dessas

\section{Gráfico 1}

Distribuição de recursos previstos pela MSS aos municípios do Rio Grande do Sul por ordem crescente de população.

\section{Curva de Lorenz}

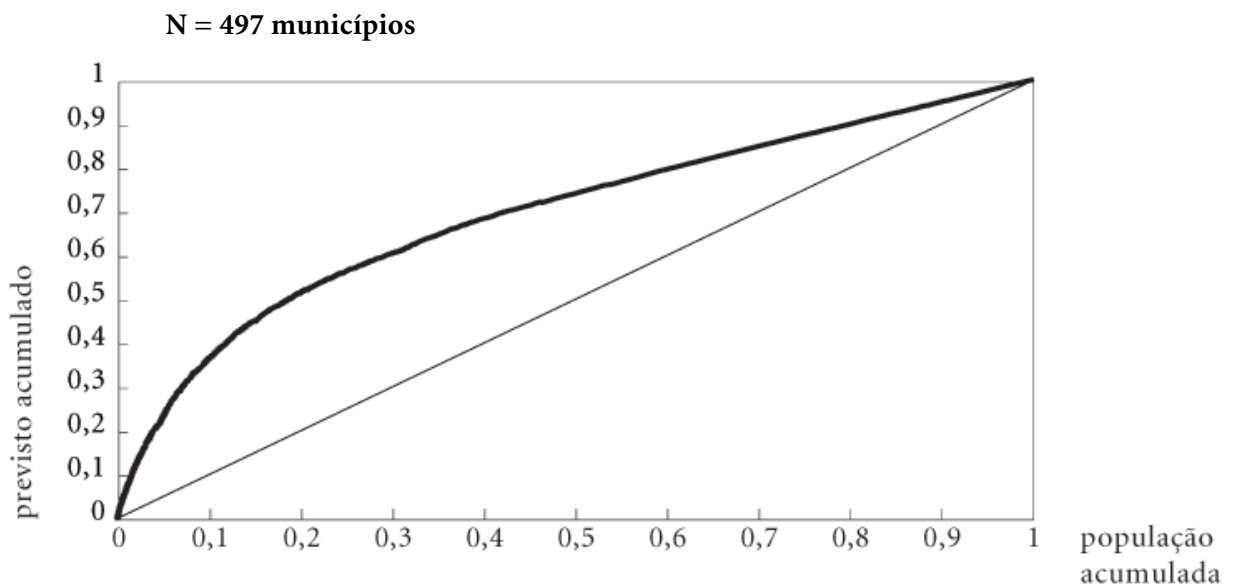




\section{Gráfico 2}

Distribuição de recursos previstos pela MSS aos municípios do Rio Grande do Sul, por ordem crescente de população residente, para municípios com menos de 10 mil habitantes, de 10.000 a 100.000 habitantes e mais de 100.000 habitantes.

\section{Curvas de Lorenz}

Municípios até 10.000 habitantes $(\mathrm{N}=338$ municípios $)$

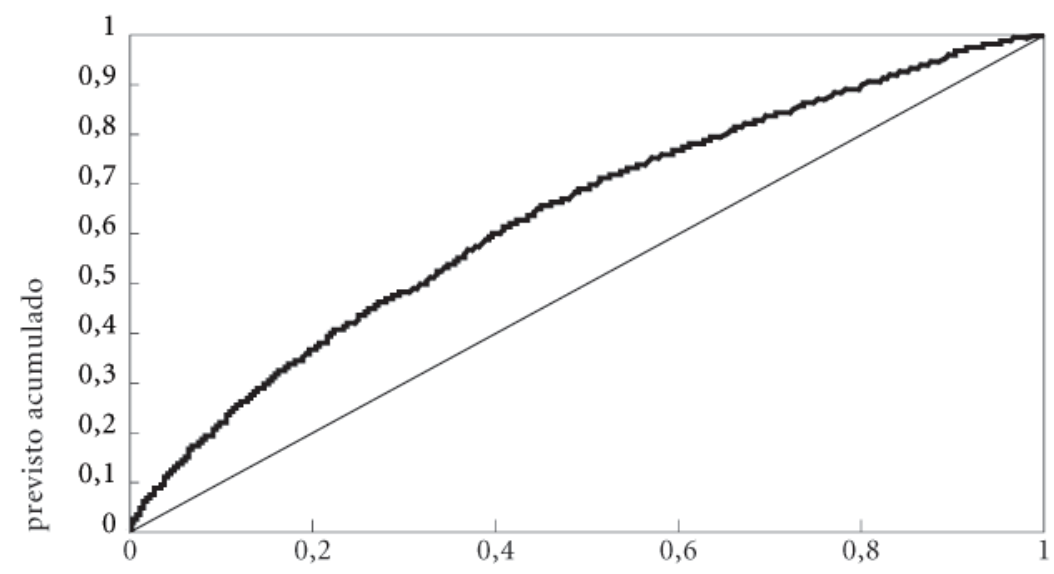

população acumulada

Municípios com 10.000 a 100.000 habitantes $(\mathrm{N}=142$ municípios $)$

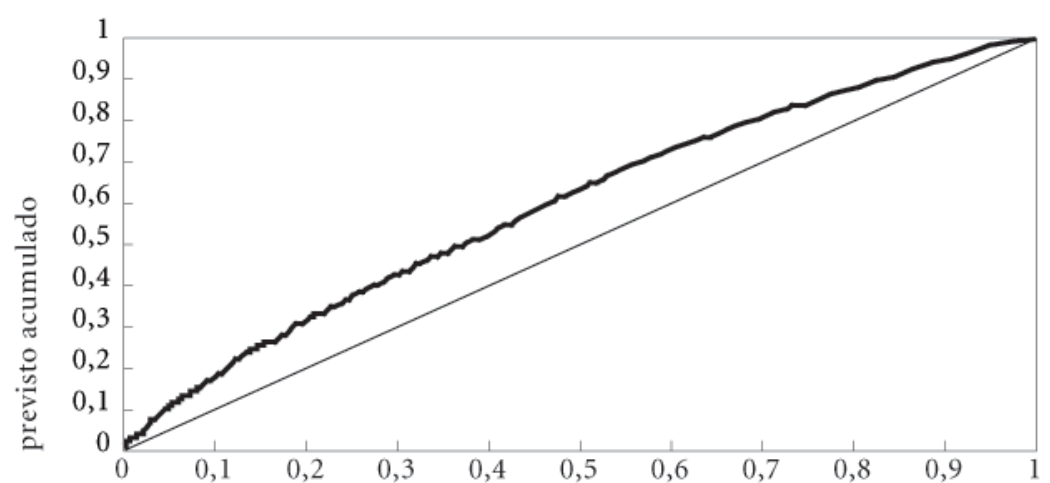

população acumulada

Municípios com mais de 100.000 habitantes ( $\mathrm{N}=17$ municípios)

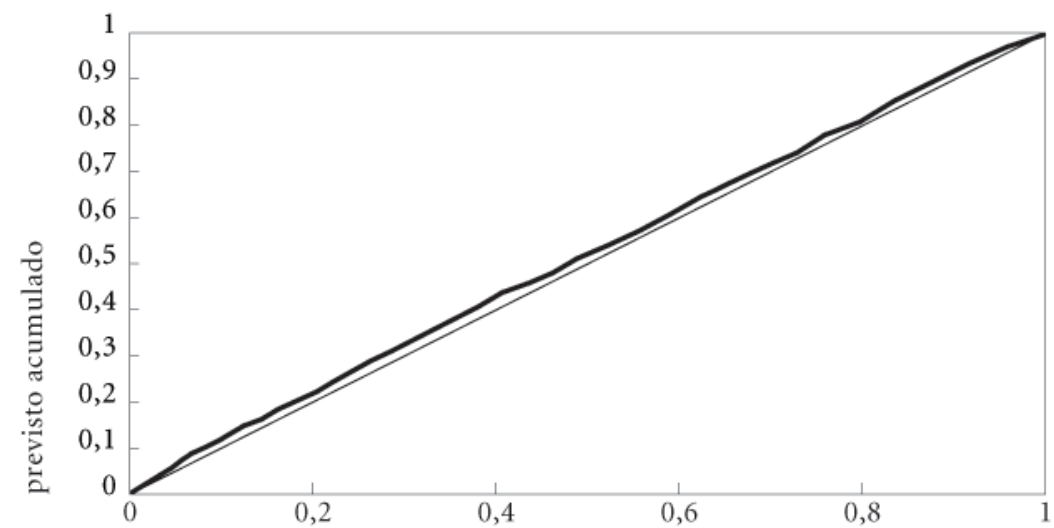

população acumulada 
categorias, foi construída a curva de Lorenz apresentada no gráfico 2 .

Ao analisar a representação gráfica do grupo de municípios com menos de 10.000 habitantes, fica evidenciado que os critérios utilizados para a distribuição de recursos estaduais, por meio da Municipalização Solidária da Saúde, tiveram capacidade discriminatória positiva para os municípios de pequeno porte, pois a curva resultante demonstra a concavidade em relação à linha de $45^{\circ}$.

Essa discriminação positiva é mantida nos municípios com população entre 10.000 e 100.000 habitantes, embora a curva observada apresente menor concavidade, se comparada com o grupo anterior. No entanto, no grupo de municípios acima de 100.000 habitantes, essa discriminação se neutraliza, obtendo-se praticamente uma linha de $45^{\circ}$, o que sinaliza uma distribuição igualitária de recursos pela população.

Esses resultados salientam a capacidade da política da MSS incidir sobre a descentralização dos recursos previstos para os municípios pequenos, o que em tese, possibilitaria um maior financiamento para custeio e investimentos em saúde nestes locais. Neste caso, um dos critérios da fórmula que mais afetou esta distribuição, e que já havia sido apontado em estudos anteriores (Ferla et al., 2003a; 2003b), foi a concentração de população maior de 60 anos nos municípios pequenos, onde é aproximadamente quatro pontos percentuais maior.

\section{Análise em relação ao nível de desenvolvimento humano municipal}

Para verificar se a Municipalização Solidária da Saúde beneficiou os municípios em piores condições de vida, analisamos através da curva de Lorenz a distribuição de recursos segundo es- ta política (MSS), com o Índice de Desenvolvimento Humano Municipal (IDH-M), conforme relatório do Programa das Nações Unidas para o Desenvolvimento (PNUD) de 2000.

O Índice de Desenvolvimento Humano Municipal é um indicador de qualidade de vida, cuja construção está baseada na metodologia do Índice de Desenvolvimento Humano (IDH), porém, com algumas modificações metodológicas nas dimensões renda e educação, necessárias para calcular um índice em nível municipal. As variáveis que compõem este índice são agrupadas em três dimensões: Renda (renda familiar per capita); Educação (obtido através da ponderação entre taxa de analfabetismo da população com 15 anos ou mais e o número médio de anos de estudos); Longevidade (expectativa de vida ao nascer). Cabe salientar que este índice agrupa os municípios em três categorias: até 0,5 - baixo desenvolvimento, até 0,8-médio desenvolvimento, acima de 0,8 - alto desenvolvimento. No Rio Grande do Sul, o relatório do PNUD identifica que existem apenas dois grupos de municípios, de médio desenvolvimento e de alto desenvolvimento.

O quadro 1 apresenta o número de municípios agrupados em IDH-M médio e alto, suas respectivas populações, valores previstos e per capita em reais. Pode-se observar que existem 307 (trezentos e nove) municípios com IDH-M médio $(0,500-0,799)$, representando $34,26 \%$ da população gaúcha com $48,60 \%$ dos recursos previstos, correspondendo a $\mathrm{R} \$ 9,13$ per capita, enquanto nos municípios de IDH-M alto (190) o valor per capita foi de $\mathrm{R} \$ 5,04$, confirmandose, assim, que os municípios com IDH-M médio receberam, proporcionalmente, um maior volume de recursos.

A curva de Lorenz, agrupando os municípios por ordem crescente de IDH-M (Gráfico 3), demonstra a desigualdade alcançada na distribuição dos recursos em favor dos municípios com IDH-M menor. Evidenciou-se que a MSS

Quadro 1

Perfil da distribuição de recursos da MSS, da população e o IDH-M dos municípios gaúchos.

\begin{tabular}{lcc}
\hline & IDH-M MÉDIO & IDH-M ALTO \\
\hline Número de municípios & 307 & 190 \\
População (habitantes) & 3.454 .561 & 6.627 .572 \\
Valores previstos em Reais & $31.556 .818,45$ & $33.373 .414,10$ \\
Per capita em Reais & 9,13 & 5,04 \\
\hline
\end{tabular}


Gráfico 3

Distribuição acumulada de recursos a municípios do Rio Grande do Sul segundo ordem crescente de IDH-M.

Curva de Lorenz

$\mathrm{N}=497$ municípios

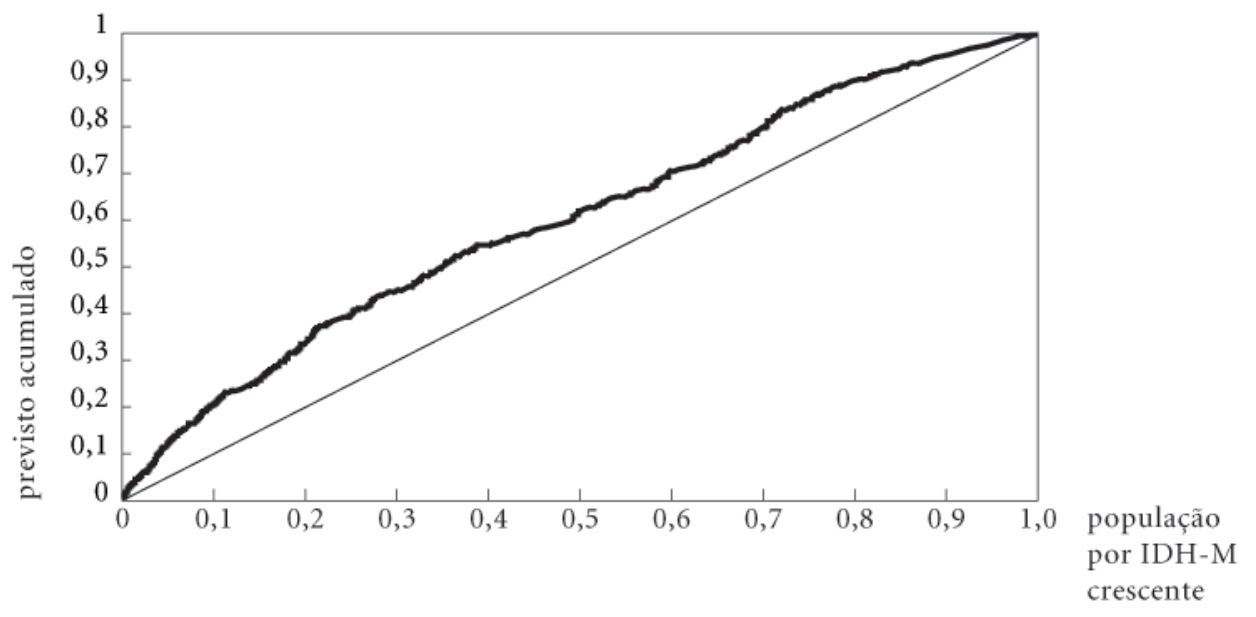

foi eqüitativa também ao considerar esse índice como avaliador das necessidades em saúde.

\section{Conclusões}

Pôde-se concluir, com essa análise, que a política de distribuição de recursos estabelecida pela MSS foi eqüitativa para as variáveis utilizadas no estudo.

É importante salientar que a desigualdade na distribuição dos recursos, evidenciada na análise realizada, com concentração dos mesmos nos municípios de menor porte, contrapõe-se às políticas até então aplicadas para o financiamento da saúde no Brasil (Barros, 2003; Carvalho, 2002), que tem historicamente aportado recursos nos grandes aglomerados urbanos, seja pela própria concentração de população (como a distribuição pelo Piso de Atenção Básica - PAB) ou pela maior oferta de procedimentos (capacidade instalada de equipamentos pagos por produtividade de serviços). Verificou-se, ainda, que os critérios de distribuição utilizados pela política de MSS têm capacidade de beneficiar os municípios com menor IDH-M.

Entretanto, é inegável a necessidade de aprofundamento da discussão conceitual e da produção de conhecimento sobre um aspecto que permeou o estudo: o impacto de fortes evidências da mudança do perfil da população para os próximos anos no conceito de eqüidade. Com o aumento da expectativa de vida, que vem sendo observado em grande número de países, particularmente na região da América Latina e do Caribe (Lebrão \& Duarte, 2003), é necessário considerar se o IDH continuará sendo um indicador com boa capacidade de auxiliar na formulação do planejamento do sistema de saúde, principalmente no que se refere ao efeito do componente "longevidade", expressando qualidade de vida. Há evidências de que a ampliação da expectativa de vida impacta no sistema de saúde, gerando a necessidade de maiores e imediatas aplicações de recursos financeiros, não só para o atendimento imediato da população que já se encontra nessas faixas etárias, como também para, rapidamente, preparar os serviços para uma aguda transição de necessidades e demandas para o setor.

Essa qualificação no conceito e nas formas de mensuração da eqüidade nas políticas sociais, em particular no planejamento da saúde, parece ser imprescindível para a construção de um sistema de saúde resolutivo, adequado às reais necessidades do seu entorno e capaz de produzir qualidade de vida, de forma crescente, para a população. 


\section{Colaboradores}

Pesquisa realizada por MLM Pelegrini para sua dissertação de mestrado, que teve JD Castro como orientadora, e ML Drachler como co-orientadora do trabalho.

\section{Referências bibliográficas}

Almeida C 2002. Eqüidade e reforma setorial na América Latina: um debate necessário. Cadernos de Saúde Pública18 (Suplemento):23-36.

Barros ME 2003. Financiamento do Sistema de Saúde no Brasil: marco legal e comportamento do gasto. Série Técnica do Projeto de Desenvolvimento de Sistemas e Serviços de Saúde/Organização Pan-Americana da Saúde, Brasília.

Barros ME 1997. Política de saúde: a complexa tarefa de enxergar a mudança onde tudo parece permanência, pp. 124-156. In AM Canesqui. Ciências sociais e saúde. Hucitec, São Paulo.

Brasil 1988. Constituição da República Federativa do Brasil. Senado Federal, Brasília.

Brasil 1990. Lei 8.080, de 19 de setembro de 1990. Dispõe sobre as condições para a promoção, proteção e recuperação da saúde, a organização e o funcionamento dos serviços de saúde correspondentes e dá outras providências. Ministério da Saúde, Brasília.

Brasil 1990. Lei 8.142, de 28 de dezembro de 1990. Dispõe sobre a participação da comunidade na gestão do Sistema Único de Saúde (SUS) e sobre as transferências intergovernamentais de recursos financeiros na área da saúde e dá outras providências. Ministério da Saúde, Brasília.

Brasil 2000. Emenda Constitucional no 29, setembro de 2000.

Briceño-León R (org.) 2000. Salud y equidad: una mirada desde las ciencias sociales. Fiocruz, Rio de Janeiro.

Carvalho GCM 2002. O financiamento público federal do Sistema Único de Saúde 1988-2001. Tese de doutorado em Saúde Pública. Faculdade de Saúde Pública, São Paulo.

Castro JD 1991. Análise da distribuição dos recursos financeiros para assistência à saúde no Brasil e no Rio Grande do Sul, uma proposta de operacionalização dos critérios para sua distribuição. Saúde em Debate 32(1):10-16

Castro JD 2002. Regulação em saúde: análise de conceitos fundamentais. Sociologias (1):122-135. UFRGS, IFCH, Porto Alegre.

Côrtes S et al. 2003. Definindo prioridades para a gestão pública: metodologia da pesquisa avaliativa de desigualdades em saúde no Rio Grande do Sul, pp. 123132. In A Ferla \& S Fagundes. Tempo de inovações: $a$ experiência da gestão na saúde do Rio Grande do Sul. Editora Da Casa, Porto Alegre.

Dachs N 2001. Inequidades en salud: como estudiarlas. In H Restrepo \& H Malaga. Promoción de la salud: como construir vida saludable. Editorial Médica Panamericana.

Drane J 1990. Cuestiones de justicia en la prestación de servicios de salud. Boletín de la Oficina Sanitaria Panamericana 108.

Faveret ACSC 2003. A vinculação constitucional de recursos para a saúde: avanços, entraves e perspectivas. Ciência \& Saúde Coletiva 8(2):371-378.

Ferla A et al. 2002. A regionalização da atenção à saúde na experiência de gestão estadual do SUS no Rio Grande do Sul, pp. 13-43. In A Ferla \& S Fagundes. O fazer em saúde coletiva: inovações da atenção à saúde no Rio Grande do Sul. Editora Da Casa, Porto Alegre.

Ferla A et al. 2003a. Atendimento integral: a escuta da gestão estadual do SUS, pp. 61-88. In R Pinheiro \& R Mattos (org.). Construção da integralidade: cotidiano, saberes e práticas em saúde. UERJ-IMS-Abrasco, Rio de Janeiro.

Ferla A et. al. 2003b. A gestão da saúde no Rio Grande do Sul no período de 1999 a 2002, pp. 247-265. In J Bittar (org.). Governos estaduais: desafios e avanços. Ed. Perseu Abramo, Rio de Janeiro.

Giraldes MR 1997. Eqüidade e despesa em saúde. Editorial Estampa, Lisboa.

Hoffmann R 1998. Estatística para economistas. (3a ed.). Ed. Pioneira, São Paulo.

IDH/2000. Índice de desenvolvimento Humano. Disponível em <http://www.undp.org.br/HDR>

Jaeger ML, Ferla A \& Pelegrini ML 2002. A gestão da saúde no contexto do SUS: descentralização, integralidade e controle social como desafios para os governos, pp. 46-85. In L Soares. Tempos de desafios. Editora Vozes, Petrópolis.

Jaeger ML, Ferla A Reichow K \& Bueno M 1999. Saúde de verdade e com qualidade: o desafio de transformar a legislação em prática cotidiana, pp. 39-50. In L Marques (org.). Rio Grande do Sul Estado e cidadania. Editora Palmarinca, Porto Alegre.

Lebrão ML \& Duarte YAO 2003. SABE - Saúde, Bem-Estar e Envelhecimento. O projeto SABE no Município de São Paulo. Organização Pan-Americana da Saúde, Brasília.

Medeiros M 1999. Princípios de justiça na alocação de recursos em saúde. Textos para Discussão no 687. IPEA, Brasília.

Mendes A \& Marques R 2002. Relatório da Oficina: "Conceitos e Instrumentos da Economia da Saúde e da Epidemiologia nos Estudos das Desigualdades". V Congresso Brasileiro de Epidemiologia, Curitiba. 
Médici AC 2002. Aspectos teóricos e conceituais do financiamento das políticas de saúde, pp. 23-68. In S Piola \& S Vianna (orgs.). Economia da saúde: conceitos e contribuição para a gestão em saúde.(3a ed.) Ipea, Brasília.

Ministério da Saúde 1991. NOB - SUS/91: Norma Operacional Básica do sistema Único de Saúde/SUS. Ministério da Saúde, Brasília.

Ministério da Saúde 1993. NOB - SUS/93: Norma Operacional Básica do Sistema Único de Saúde/SUS. Ministério da Saúde, Brasília.

Ministério da Saúde 1997. NOB - SUS/96: Norma Operacional Básica do Sistema Único de Saúde/SUS. Ministério da Saúde, Brasília.

Ministério da Saúde 2002. Experiências inovadoras no SUS: gestão dos serviços de saúde. Vol. 1. Ministério da Saúde, Brasília.

Pastrana R \& Cunha F 2002. O Sistema de Informações sobre Orçamentos Públicos em Saúde - Siops: instrumento de gestão e controle social. VI Encontro Nacional de Economia de Saúde - ABRES, CD-ROM. Rio de Janeiro.

Porto S 2002. Justiça, eqüidade e necessidades em saúde. Tese de doutorado. Escola Nacional de Saúde Pública, Rio de Janeiro.

Rio Grande do Sul 1989. Constituição do Estado do Rio Grande do Sul. Diário Oficial do Estado. Porto Alegre.

Rio Grande do Sul 1999. Decreto Estadual no 39.582. Diário Oficial do Estado. Porto Alegre. 10 junho de 1999.

Rounet LP 2003. Justiça como eqüidade: uma proposta brasileira. Niterói, jul.2002. Disponível em: $<$ http:// www.cienciapolitica.org.br/encontro/teopol4.3.doc $>$. Acesso em 12 de janeiro, 2003.
Secretaria da Saúde do Rio Grande do Sul 1999. Portaria SES/RS № 09/99. 21 de junho de 1999.

Secretaria da Saúde do Rio Grande do Sul 2001. Portaria SES/RS № 26/2001. 29 de junho de 2001.

Sen A 2002a. Sobre ética e economia. Trad. Laura Teixeira Motta. Ed. Companhia das Letras, São Paulo.

Sen A 2002b. Desenvolvimento como liberdade. Trad. Laura Teixeira Motta. Ed. Companhia das Letras, São Paulo.

Teixeira HV \& Teixeira MG 2003. Financiamento da saúde no Brasil: a experiência do SIOPS. Ciência \& Saúde Coletiva 8(2):379-391.

Tobar F et al. 2003. Modelos eqüitativos de distribuição de recursos sanitários. Disponível em <htpp://www.opas. org.br/serviços/Arquivos/sala5205.pdf $>$. Acesso em 5 de maio, 2003.

Ugá MA, Piola S, Porto S \& Vianna S 2003. Descentralização e alocação de recursos no âmbito do SUS. Ciência \& Saúde Coletiva 8(2):417-437.

Vianna S et al. 1991. O financiamento da descentralização dos serviços de saúde: critérios para transferências de recursos federais para estados e municípios. Ipea, Brasília.

Vianna S 2002. Revisando a distribuição de encargos na saúde entre as esferas de governo. PROJETO BRA/97/ 013. VI Encontro Nacional de Economia de SaúdeABRES, CD-ROM. Rio de Janeiro.

Vianna S et al. 2001. Medindo as desigualdades em saúde no Brasil: uma proposta de monitoramento. Organização Pan-Americana da Saúde. Ipea, Brasília. 